\title{
Exact Approach to Uniform Time-Varying Magnetic Field
}

\author{
Mauro Bologna (1) \\ Instituto de Alta Investigación, Universidad de Tarapacá, Casilla 6-D, Arica, Chile \\ Correspondence should be addressed to Mauro Bologna; mauroh69@gmail.com
}

Received 3 August 2018; Revised 24 September 2018; Accepted 1 October 2018; Published 16 October 2018

Academic Editor: Jan Koci

Copyright (C) 2018 Mauro Bologna. This is an open access article distributed under the Creative Commons Attribution License, which permits unrestricted use, distribution, and reproduction in any medium, provided the original work is properly cited.

In this paper, we will find in terms of Fourier integrals an exact expression for the magnetic and electric fields generated by an infinite solenoid where an arbitrary current density $\mathbf{J}(t)$ flows. Considering the central region of an infinite solenoid, we will obtain an exact expression relating the flowing current and the magnetic and electric fields as functions of the time. Being an exact expression, it will allow us to go beyond the quasistatic approximation. The result can apply in theoretical problems and experimental setups where the flowing current may change abruptly in time, such as pulsed or stochastic currents.

\section{Introduction}

In physics and engineering uniform magnetic fields are ubiquitous. Many studies are devoted about how to generate them. For this purpose, Helmholtz coils are widely used but new designs to create uniform fields are present in literature [1-3]. For example, uniform fields are used in magnetohydrodynamic studies [4-8], in ferrofluid flow studies [9], in new analytical approaches to the studies of micropolar conducting ferrofluids with applications to new engineering materials $[10,11]$, in magnetic resonance spectroscopy [12], in technological applications $[13,14]$, in testing devices [15], or in measuring instruments such as magnetometers. Strictly related to the previous topic we also consider time-varying uniform magnetic fields that are widely used in theoretical and experimental works such as magnetic field susceptibility tests or biomedical studies [16-18]. The literature on this topic is quite vast, and we limit ourselves to a few exemplary applicative papers [19-22]. The typical approach is based on the quasistatic approximation where the magnetic field $\mathbf{H}(t)$ is given, in the SI system of units, by the flowing current density $\mathbf{J}(t)$, i.e., $\mathbf{H}(t) \approx \mathbf{J}(t)$. If we consider arbitrary timevarying currents, then things start to be a little more complicated. Via Maxwell's equations, we know that an electric field is created, according to the Lenz's law [23, 24]. We focus on which kind of magnetic and electric fields we have to consider when the first order approximation is a uniform magnetic field varying arbitrarily in time. This can be realized by studying the electromagnetic field generated by an infinite solenoid where an arbitrary time-varying current density $\mathbf{J}(t)$ flows. To the best of our knowledge, from an analytical point of view, the generation of an electromagnetic field by an infinite solenoid is not completely solved except for particular cases $[25,26]$. The knowledge of the central values of the fields will allow us to go beyond the quasistatic approximation and we may apply the results to actual laboratory setups. For example, circuits with abruptly changing currents, such as an impulse current generator circuit, are currently used in electrical engineering. Another important case, where the quasistatic approximation loses validity due to the abrupt changes of the current, is a magnetic field generated by a stochastic current, a well-known phenomenon since a long time ([27] and references therein). With respect to these cases, the first-order approximation of a uniform magnetic field proportional to the flowing current is not sufficient since the magnetic and the induced electric fields are related to the derivatives of the flowing current.

Let us clarify this crucial point considering the set of Maxwell's equations [23, 24] in the empty space

$$
\begin{aligned}
& \nabla \times \mathbf{E}=-\frac{\partial \mathbf{B}}{\partial t}, \\
& \nabla \times \mathbf{H}=\frac{\partial \mathbf{D}}{\partial t},
\end{aligned}
$$




$$
\begin{aligned}
& \nabla \cdot \mathbf{E}=0, \\
& \nabla \cdot \mathbf{B}=0,
\end{aligned}
$$

where $\mathbf{E}$ is the electric field, $\mathbf{D}$ is the electric displacement field related to the electric field $\mathbf{E}$ via the relationship $\mathbf{D}=\varepsilon_{0} \mathbf{H}$, and $\mathbf{B}$ is the magnetic induction, related to the magnetic field $\mathbf{H}$ via the relationship $\mathbf{B}=\mu_{0} \mathbf{H}$. The constant $\varepsilon_{0}=8.85 \times 10^{-12}$ farads/meter is the vacuum permittivity in the SI system of units. The constant $\mu_{0}=4 \pi \times 10^{-7}=1.256637061 \times 10^{-6}$ henries/meter is the vacuum permeability in the SI system of units.

If we solve iteratively the Maxwell equations we may assume that a slow time-varying current generates a magnetic induction field $\mathbf{B}=\mathbf{B}_{0}(t)+\delta \mathbf{B}$ and an electric field $\mathbf{E}=\delta \mathbf{E}$ where $\mathbf{B}_{0}(t)$ is the uniform field associated with the current density, i.e., $\mathbf{B}_{0}(t)=\mu_{0} \mathbf{J}(t)$, and $\delta \mathbf{B}, \delta \mathbf{E}$ are the corrections. At first order, in the vacuum space, for (1) and (2) we have

$$
\begin{gathered}
\nabla \times \delta \mathbf{E}=-\frac{\partial}{\partial t} \mathbf{B} \approx-\frac{\partial}{\partial t} \mathbf{B}_{\mathbf{0}}(t), \\
\nabla \times \mathbf{H}=\nabla \times \delta \mathbf{H}=\frac{\partial}{\partial t} \delta \mathbf{D} .
\end{gathered}
$$

From (5) and (6) we deduce that iterating this approach, we end up into time derivatives of the fields of arbitrary order. This fact can invalidate the above approach in presence of an arbitrary time-varying current $\mathbf{J}(t)$. Indeed considering the case when the current changes abruptly, then the iterative approach would give rise to very large derivatives of the fields, forcing us to take into account all subsequent derivative orders. A simple and realistic example is a dichotomously fluctuating current. The derivatives of the fields would produce Dirac's delta functions and its derivatives. These considerations imply that a simple perturbative-iterative approach is not feasible for the problem that we are studying.

The paper is organized as follows: In Section 2 we will evaluate an exact expression, in terms of Fourier transform, for the magnetic and electric fields generated by an arbitrary current density flowing on the surface of a solenoid. In Section 3 we will apply the result to a harmonic current density flowing on the surface of the solenoid obtaining an exact expression for the fields inside and outside of the solenoid. In Section 4 we will evaluate the exact expression of magnetic and electric fields, in time representation, at the center of the solenoid. The obtained expressions take into account the correction to the quasistatic approach giving an analytical expression for the fields generated by an arbitrary time-varying current density. Finally in Section 5 we will draw the final remarks.

\section{Infinite Solenoid Field}

In this section, we will evaluate the exact expression for the magnetic and electric field generated by an arbitrary current density flowing on the surface of an infinite solenoid of radius $a$ (see Figure 1). In presence of arbitrary currents and zero

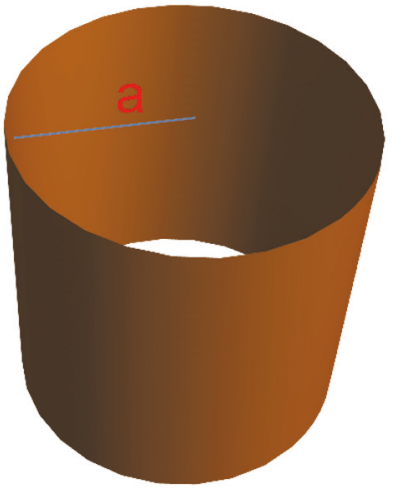

FIGURE 1: Solenoid of radius $a$. On its surface a uniform current density $\mathbf{J}(t)=I(t) \widehat{\boldsymbol{\theta}}$ flows.

charge density, the general solution of (1)-(4) is given in terms of the potential vector $\mathbf{A}(\mathbf{x}, t)$ (Coulomb gauge [24])

$$
\mathbf{A}(\mathbf{x}, t)=\frac{\mu_{0}}{4 \pi} \int_{V} \frac{\mathbf{J}\left(\mathbf{x}^{\prime}, t^{\prime}\right)}{\left|\mathbf{x}-\mathbf{x}^{\prime}\right|} d^{3} x^{\prime}
$$

where $d^{3} x^{\prime}$ is the volume element at $\mathbf{x}^{\prime}$, the current density $\mathbf{J}$ is a given function of space and time, and the time $t^{\prime}$ is given by the relation [24]

$$
t^{\prime}=t-\frac{|\mathbf{x}|}{c}
$$

We may rewrite (7) as

$$
\begin{aligned}
& \mathbf{A}(\mathbf{x}, t)=\frac{\mu_{0}}{4 \pi} \int_{-\infty}^{\infty} \int_{V} \frac{\mathbf{J}\left(\mathbf{x}^{\prime}, \omega\right)}{\left|\mathbf{x}-\mathbf{x}^{\prime}\right|} \exp \left[-\imath \omega t^{\prime}\right] \frac{d \omega}{2 \pi} d^{3} x^{\prime} \\
& =\frac{\mu_{0}}{4 \pi} \int_{-\infty}^{\infty} \int_{V} \frac{\mathbf{J}\left(\mathbf{x}^{\prime}, \omega\right)}{\left|\mathbf{x}-\mathbf{x}^{\prime}\right|} \exp \left[-\imath \omega\left(t-\frac{\left|\mathbf{x}-\mathbf{x}^{\prime}\right|}{c}\right)\right] \\
& \quad \cdot \frac{d \omega}{2 \pi} d^{3} x^{\prime} .
\end{aligned}
$$

Knowing the potential vector we may evaluate the magnetic induction and electric fields via the equations

$$
\begin{aligned}
& \mathbf{B}(\mathbf{x}, t)=\nabla \times \mathbf{A}(\mathbf{x}, t), \\
& \mathbf{E}(\mathbf{x}, t)=-\frac{\partial}{\partial t} \mathbf{A}(\mathbf{x}, t) .
\end{aligned}
$$

Considering as system an infinite solenoid, we have two empty spaces separated by the surface of the solenoid where the current is flowing. If we consider the Fourier representation of (7), we may write that

$$
\widehat{\mathbf{A}}(\mathbf{x}, \omega)=\frac{\mu_{0}}{4 \pi} \int_{V} \frac{\widehat{\mathbf{J}}(\mathbf{x}, \omega)}{\left|\mathbf{x}-\mathbf{x}^{\prime}\right|} \exp \left[\imath \frac{\omega}{c}\left|\mathbf{x}-\mathbf{x}^{\prime}\right|\right] d^{3} x^{\prime}
$$


where we defined

$$
\begin{aligned}
\mathbf{A}(\mathbf{x}, t) & =\int_{-\infty}^{\infty} \widehat{\mathbf{A}}(\mathbf{x}, \omega) \exp [-\imath \omega t] \frac{d \omega}{2 \pi}, \\
\mathbf{J}(\mathbf{x}, t) & =\int_{-\infty}^{\infty} \widehat{\mathbf{J}}(\mathbf{x}, \omega) \exp [-\imath \omega t] \frac{d \omega}{2 \pi} .
\end{aligned}
$$

Taking into account the fact that $\mathbf{J}$ is a two-dimensional current density, we have that $d^{3} x^{\prime} \longrightarrow a d \theta^{\prime} d z^{\prime}$ where $a$ is the solenoid radius. For the sake of simplicity, we consider a uniform current density in the angular direction, i.e., $\widehat{\mathbf{J}}(\mathbf{x}, \omega)=\widehat{I}_{\omega} \widehat{\boldsymbol{\theta}}$, and we obtain

$$
\begin{aligned}
& \widehat{\mathbf{A}}(\mathbf{x}, \omega) \\
& =\frac{\mu_{0} a \widehat{I}_{\omega}}{4 \pi} \int_{-\infty}^{\infty} \int_{0}^{2 \pi} \frac{\exp \left[\imath(\omega / c)\left|\mathbf{x}-\mathbf{x}^{\prime}\right|\right]}{\left|\mathbf{x}-\mathbf{x}^{\prime}\right|} \widehat{\boldsymbol{\theta}}^{\prime} d z^{\prime} d \theta^{\prime} .
\end{aligned}
$$

Setting $u=\sqrt{a^{2}+r^{2}-2 a r \cos \left(\theta-\theta^{\prime}\right)}$ we may rewrite (15) as

$$
\begin{aligned}
& \widehat{\mathbf{A}}(\mathbf{x}, \omega) \\
& \quad=\frac{\mu_{0} a \widehat{I}_{\omega}}{4 \pi} \int_{-\infty}^{\infty} \int_{0}^{2 \pi} \frac{\exp \left[\imath(\omega / c) \sqrt{z^{\prime 2}+u^{2}}\right]}{\sqrt{z^{\prime 2}+u^{2}}} \widehat{\boldsymbol{\theta}}^{\prime} d z^{\prime} d \theta^{\prime} .
\end{aligned}
$$

To compact the notation, we define the following quantities:

$$
\begin{aligned}
& a^{*}=\frac{a}{c}, \\
& r^{*}=\frac{r}{c}, \\
& u^{*}=\sqrt{a^{* 2}+r^{* 2}-2 a^{*} r^{*} \cos \left(\theta-\theta^{\prime}\right)},
\end{aligned}
$$

and we will dropped the space and frequency dependance of the field $\widehat{\mathbf{A}}(\mathbf{x}, \omega)$. Performing the integration on the $z^{\prime}$ variable and projecting along the $x, y$ axes we obtain

$$
\begin{aligned}
& \widehat{A}_{x}=\frac{\mu_{0} c a^{*} \widehat{I}_{\omega}}{4} \\
& \cdot \int_{0}^{2 \pi}\left[Y_{0}\left(|\omega| u^{*}\right)-\imath \operatorname{sgn}(\omega) J_{0}\left(|\omega| u^{*}\right)\right] \sin \theta^{\prime} d \theta^{\prime}, \\
& \widehat{A}_{y}=-\frac{\mu_{0} c a^{*} \widehat{I}_{\omega}}{4} \\
& \cdot \int_{0}^{2 \pi}\left[Y_{0}\left(|\omega| u^{*}\right)-\imath \operatorname{sgn}(\omega) J_{0}\left(|\omega| u^{*}\right)\right] \\
& \cdot \cos \theta^{\prime} d \theta^{\prime}
\end{aligned}
$$

where $J_{0}(x)$ and $Y_{0}(x)$ are the zero order Bessel functions of the first and second kind, respectively, while $\operatorname{sgn}(\omega)$ is the function that gives the sign of $\omega$. Using Graf's addition theorem for Bessel functions $[28,29]$ we have

$$
\begin{gathered}
\exp [\imath \imath \psi] Z_{\nu}\left(k u^{*}\right) \\
=\sum_{n=-\infty}^{\infty} J_{n}\left(k r_{<}\right) Z_{\nu+n}\left(k r_{>}\right) \exp [\imath n \phi], \\
\quad \phi \equiv \theta-\theta^{\prime}, \\
\exp [2 \imath \psi]=\frac{r^{*}-a^{*} \exp [-\imath \phi]}{r^{*}-a^{*} \exp [\imath \phi]}, \quad 0<\psi<\frac{\pi}{2},
\end{gathered}
$$

where $k$ is an arbitrary complex number, $r_{<}=\min \left(a^{*}, r^{*}\right)$ and $r_{>}=\max \left(a^{*}, r^{*}\right), Z_{\nu}(x)$ is any of the Bessel functions $J_{\nu}(x), Y_{\nu}(x), I_{\nu}(x), K_{\nu}(x)$ or the Hankel functions of the first and second kind, $H_{v}^{(1)}(x)$ and $H_{v}^{(2)}(x)$, respectively. Let us focus on the solution inside to the solenoid. Then $r_{<}=r^{*}$ and $r_{>}=a^{*}$; thus we have

$$
\begin{aligned}
\widehat{A}_{x} & =\frac{\mu_{0} c a^{*} \widehat{I}_{\omega} \pi}{2} \times\left[Y_{1}\left(|\omega| a^{*}\right)-\imath \operatorname{sgn}(\omega) J_{1}\left(|\omega| a^{*}\right)\right] \\
\cdot & J_{1}\left(|\omega| r^{*}\right) \sin \theta \\
\widehat{A}_{y} & =-\frac{\mu_{0} c a^{*} \widehat{I}_{\omega} \pi}{2} \\
& \times\left[Y_{1}\left(|\omega| a^{*}\right)-\imath \operatorname{sgn}(\omega) J_{1}\left(|\omega| a^{*}\right)\right] J_{1}\left(|\omega| r^{*}\right) \\
\cdot & \cos \theta
\end{aligned}
$$$$
\text { or in polar coordinates }
$$

$$
\begin{aligned}
& \widehat{A}_{\theta}=-\frac{\mu_{0} c a^{*} \widehat{I}_{\omega} \pi}{2}\left[Y_{1}\left(|\omega| a^{*}\right)-\imath \operatorname{sgn}(\omega) J_{1}\left(|\omega| a^{*}\right)\right] \\
& \cdot J_{1}\left(|\omega| r^{*}\right) .
\end{aligned}
$$

Exploiting the relationship $\mathbf{B}=\mu_{0} \mathbf{H}$ we obtain the magnetic field in the inner region of the solenoid via the Fourier transform of (10), i.e.,

$$
\begin{aligned}
\widehat{H}_{z}^{i n}= & \frac{1}{\mu_{0}} \widehat{B}_{z}^{i n}=\frac{1}{\mu_{0} r} \frac{\partial}{\partial r}\left(r \widehat{A}_{\theta}\right) \\
= & -\frac{\widehat{I}_{\omega} \pi}{2}|\omega| a^{*} \\
& \times\left[Y_{1}\left(|\omega| a^{*}\right)-\imath \operatorname{sgn}(\omega) J_{1}\left(|\omega| a^{*}\right)\right] J_{0}\left(\omega r^{*}\right) .
\end{aligned}
$$

Similarly the electric field, via the Fourier transform of (11), is

$$
\begin{aligned}
\widehat{E}_{\theta}^{i n}= & \imath \omega \widehat{A}_{\theta} \\
= & -\imath \frac{\mu_{0} c \widehat{I}_{\omega} \pi}{2}|\omega| a^{*} \\
& \times\left[Y_{1}\left(|\omega| a^{*}\right)-\imath \operatorname{sgn}(\omega) J_{1}\left(|\omega| a^{*}\right)\right] J_{1}\left(\omega r^{*}\right) .
\end{aligned}
$$


Going back to the time representation we may write

$$
\begin{aligned}
& H_{z}^{\text {in }}(r, t)=-\frac{a^{*} \pi}{2} \int_{-\infty}^{\infty} \frac{d \omega}{2 \pi} \widehat{I}_{\omega}|\omega| \\
& \cdot\left[Y_{1}\left(|\omega| a^{*}\right)-\imath \operatorname{sgn}(\omega) J_{1}\left(|\omega| a^{*}\right)\right] J_{0}\left(\omega r^{*}\right) \\
& \cdot \exp [-\imath \omega t], \\
& E_{\theta}^{\text {in }}(r, t)=-\imath \frac{\mu_{0} c a^{*} \pi}{2} \int_{-\infty}^{\infty} \frac{d \omega}{2 \pi} \widehat{I}_{\omega}|\omega| \\
& \cdot\left[Y_{1}\left(|\omega| a^{*}\right)-\imath \operatorname{sgn}(\omega) J_{1}\left(|\omega| a^{*}\right)\right] J_{1}\left(\omega r^{*}\right) \\
& \cdot \exp [-\imath \omega t] .
\end{aligned}
$$

In a similar way, we can evaluate the magnetic and electric fields in the region outside of the solenoid. In this case we find

$$
\begin{aligned}
& H_{z}^{\text {out }}(r, t)=-\frac{a^{*} \pi}{2} \int_{-\infty}^{\infty} \frac{d \omega}{2 \pi} \exp [-\imath \omega t] \widehat{I}_{\omega}|\omega| \\
& \quad \times\left[J_{1}\left(|\omega| a^{*}\right) Y_{0}\left(|\omega| r^{*}\right)\right. \\
& \left.\quad-\imath \operatorname{sgn}(\omega) J_{1}\left(|\omega| a^{*}\right) J_{0}\left(\omega r^{*}\right)\right], \\
& E_{\theta}^{\text {out }}(r, t)=-\imath \frac{\mu_{0} c a^{*} \pi}{2} \int_{-\infty}^{\infty} \frac{d \omega}{2 \pi} \exp [-\imath \omega t] \widehat{I}_{\omega} \omega \\
& \quad \times\left[J_{1}\left(|\omega| a^{*}\right) Y_{1}\left(|\omega| r^{*}\right)\right. \\
& \left.-\imath \operatorname{sgn}(\omega) J_{1}\left(|\omega| a^{*}\right) J_{1}\left(|\omega| r^{*}\right)\right] .
\end{aligned}
$$

\section{Exact Case: Harmonic Current Density}

As an exact example, we will consider the case of a harmonic current density flowing on the solenoid surface along the angular direction $\widehat{\boldsymbol{\theta}}$. We assume that

$$
\mathbf{J}(t)=I(t) \hat{\boldsymbol{\theta}}=I_{0} \cos \left(\omega_{0} t+\phi\right) \hat{\boldsymbol{\theta}} .
$$

Taking the Fourier transform we have

$$
\begin{aligned}
& \widehat{I}_{\omega} \\
& \quad=I_{0} \pi\left[\exp [\imath \phi] \delta\left(\omega+\omega_{0}\right)+\exp [-\imath \phi] \delta\left(\omega-\omega_{0}\right)\right] .
\end{aligned}
$$

From (28) and (29) it follows that

$$
\begin{aligned}
H_{z}^{i n}(r, t) & \\
=- & \frac{\pi}{2} I_{0} \omega_{0} a^{*} Y_{1}\left(\omega_{0} a^{*}\right) J_{0}\left(\omega_{0} r^{*}\right) \cos \left(\omega_{0} t+\phi\right) \\
& +\frac{\pi}{2} I_{0} \omega_{0} a^{*} J_{1}\left(\omega_{0} a^{*}\right) J_{0}\left(\omega_{0} r^{*}\right) \sin \left(\omega_{0} t+\phi\right) . \\
E_{\theta}^{i n}(r, t) & -\frac{\mu_{0} c \pi}{2} I_{0} \omega_{0} a^{*} Y_{1}\left(\omega_{0} a^{*}\right) J_{1}\left(\omega_{0} r^{*}\right) \sin \left(\omega_{0} t+\phi\right) \\
\quad & \frac{\mu_{0} c a^{*} \pi}{2} I_{0} \omega_{0} J_{1}\left(\omega_{0} a^{*}\right) J_{1}\left(\omega_{0} r^{*}\right) \cos \left(\omega_{0} t+\phi\right) .
\end{aligned}
$$

We shall focus now on the internal magnetic field, i.e., $H_{z}^{i n}(r, t)$. For $\omega_{0} a^{*} \ll 1$ and consequently $\omega_{0} r^{*} \ll 1$, we may approximate the Bessel functions as

$$
\begin{aligned}
& J_{0}\left(\omega_{0} r^{*}\right) \approx 1 \\
& Y_{1}\left(\omega_{0} a^{*}\right) \approx-\frac{2}{\pi \omega_{0} a^{*}}, \\
& J_{1}\left(\omega_{0} a^{*}\right) \approx \frac{\omega_{0} a^{*}}{2}
\end{aligned}
$$

Thus the current density $I(t)=I_{0} \cos \left(\omega_{0} t+\phi\right)$ is a slow timevarying function and we recover the well-known result

$$
\begin{aligned}
H_{z}^{i n}(r, t) & \approx I_{0} \cos \left(\omega_{0} t+\phi\right), \\
E_{\theta}^{i n}(r, t) & \approx \frac{r^{*}}{2} \mu_{0} c \omega_{0} I_{0} \sin \left(\omega_{0} t+\phi\right) \\
& =-\frac{r}{2} \frac{\partial}{\partial t} B_{z}^{i n}(r, t) .
\end{aligned}
$$

In the opposite limit, i.e., $\omega_{0} a^{*} \gg 1$, we may distinguish two cases: on the axis and on the solenoid surface. On the axis we have

$$
H_{z}^{i n}(0, t)=-I_{0} \sqrt{\frac{\pi \omega_{0} a^{*}}{2}} \sin \left[\omega_{0}\left(t-a^{*}\right)+\phi-\frac{\pi}{4}\right]
$$

while on the solenoid surface we have

$$
\begin{aligned}
& H_{z}^{i n}(a, t) \\
& =-I_{0} \sqrt{\frac{\pi \omega_{0} a^{*}}{2}} \sin \left[\omega_{0}\left(t-a^{*}\right)+\phi-\frac{\pi}{4}\right] J_{0}\left(\omega_{0} a^{*}\right) \\
& \approx-I_{0} \sin \left[\omega_{0}\left(t-a^{*}\right)+\phi-\frac{\pi}{4}\right] \cos \left(\omega_{0} a^{*}-\frac{\pi}{4}\right) .
\end{aligned}
$$

As we can infer from (34), there are frequencies at which the magnetic field evaluated on the surface vanishes (see Figures 2 and 3). Such frequencies are given by the zeros of $J_{0}\left(\omega_{0} a^{*}\right)$ that in the high-frequency limit are

$$
\omega_{0}(n) a^{*}=\frac{\pi}{4}+(2 n+1) \frac{\pi}{2}, \quad \text { for } n \gg 1
$$

On the other hand the amplitude of the central value of the magnetic field grows as $\sqrt{\omega_{0} a^{*}}$. In a similar way, from (35) we deduce that the frequencies, at which the electric field $E_{\theta}^{i n}(r, t)$ evaluated on the surface vanishes, are given by the zero of $J_{1}\left(\omega_{0} a^{*}\right)$, i.e.,

$$
\omega_{0}(n) a^{*}=-\frac{\pi}{4}+(2 n+1) \frac{\pi}{2}, \quad \text { for } n \gg 1 \text {. }
$$


Analogous calculation are performed for the external fields. Using (30) and (31), for the magnetic and electric fields we have

$$
\begin{gathered}
H_{z}^{\text {out }}(r, t)=-\frac{a^{*} \pi}{2} I_{0} \omega_{0} J_{1}\left(\omega_{0} a^{*}\right) \\
\cdot\left[Y_{0}\left(\omega_{0} r^{*}\right) \cos \left(\omega_{0} t+\phi\right)\right. \\
\left.-J_{0}\left(\omega_{0} r^{*}\right) \sin \left(\omega_{0} t+\phi\right)\right], \\
E_{\theta}^{\text {out }}(r, t)=-\frac{\mu_{0} c a^{*} \pi}{2} I_{0} \omega_{0} J_{1}\left(\omega_{0} a^{*}\right) \\
\cdot\left[Y_{1}\left(\omega_{0} r^{*}\right) \sin \left(\omega_{0} t+\phi\right)\right. \\
\left.+J_{1}\left(\omega_{0} r^{*}\right) \cos \left(\omega_{0} t+\phi\right)\right] .
\end{gathered}
$$

An easy check shows that for $\omega_{0} a^{*} \ll 1$ we recover the quasistatic limit $H_{z}^{\text {out }}(r, t), E_{\theta}^{\text {out }}(r, t) \approx 0$. Finally using the Bessel function identity $Y_{1}(z) J_{0}(z)-Y_{0}(z) J_{1}(z)=-2 / \pi z$, it is straightforward to verify that $\mathbf{H}$ satisfies to the boundary condition

$$
H_{z}^{\text {in }}(a, t)-H_{z}^{\text {out }}(a, t)=I_{0} \cos \left(\omega_{0} t+\phi\right)=I(t) .
$$

A remarkable property of (43) and (44) is that there is a series of frequencies at which both magnetic and electric fields vanish outside of the solenoid. Indeed having the expression of the fields the common factor $J_{1}\left(\omega_{0} a^{*}\right)$, we find that the outside fields vanish when

$$
\begin{aligned}
& J_{1}\left(\omega_{0} a^{*}\right)=0, \Longrightarrow \\
& \omega_{0}(n) a^{*} \approx(2 n+1) \frac{\pi}{2}-\frac{\pi}{4}, \text { for } n \gg 1 .
\end{aligned}
$$

The first exact frequency at which the fields $H_{z}^{\text {out }}(r, t)$ and $E_{\theta}^{\text {out }}(r, t)$ vanish is given by the first zero of $J_{1}(z)$ corresponding to $\omega_{0}(1) a^{*}=3.83171$. The results are shown visually in Figures 4 and 5 .

\section{Axial Fields}

In this section, we will evaluate the magnetic and electric field at the center of the solenoid. The central region of the solenoid is the region where the magnetic field can be considered uniform. Being evaluated using an exact expression, we will have the field values that are consistent with Maxwell's equations. For the sake of simplicity, we will set the origin of time at $t=0$. In other words, there are no currents flowing for $t<0$. We will find a generic expression for the field $\mathbf{H}$ and E. To do that we consider (28) evaluated at $r=0$. We have

$$
\begin{aligned}
H_{z}^{i n}(0, t)=-\frac{a^{*} \pi}{2} \int_{-\infty}^{\infty} \frac{d \omega}{2 \pi} \widehat{I}_{\omega}|\omega| \\
\cdot\left[Y_{1}\left(|\omega| a^{*}\right)-\imath J_{1}\left(\omega a^{*}\right)\right] \exp [-\imath \omega t], \quad t>0 .
\end{aligned}
$$

Applying the properties of Fourier transform we may rewrite the above expression as

$$
H_{z}^{i n}(0, t)=-\frac{a^{*} \pi}{2} \int_{-\infty}^{\infty} I\left(t-t^{\prime}\right) G\left(t^{\prime}\right) d t^{\prime}
$$

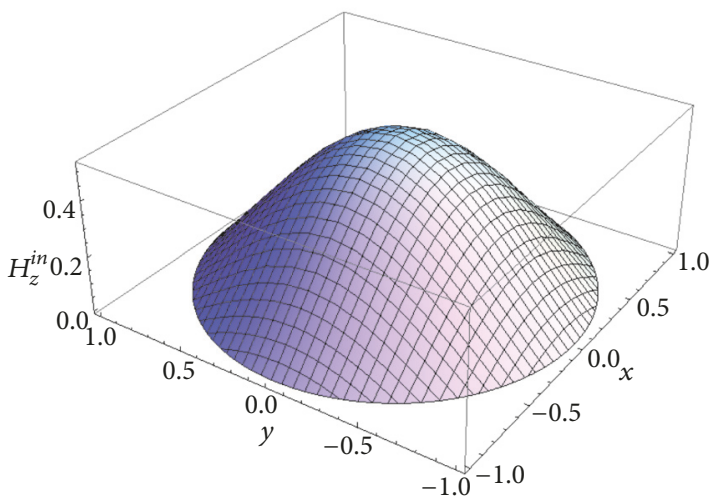

FIGURE 2: Plot of the magnetic field $H_{z}^{i n}(r, t)$ (see (34)) as function of the radial distance from the solenoid center $r=\sqrt{x^{2}+y^{2}}$. The parameters $I_{0}$ and $a^{*}$ have been set equal to the unit, while the field is evaluated at $\omega_{0} t=\pi / 4$ and $\omega_{0}=2.4048$, i.e., the first zero of $J_{0}(z)$. At this frequency the magnetic field vanishes on the solenoid surface.

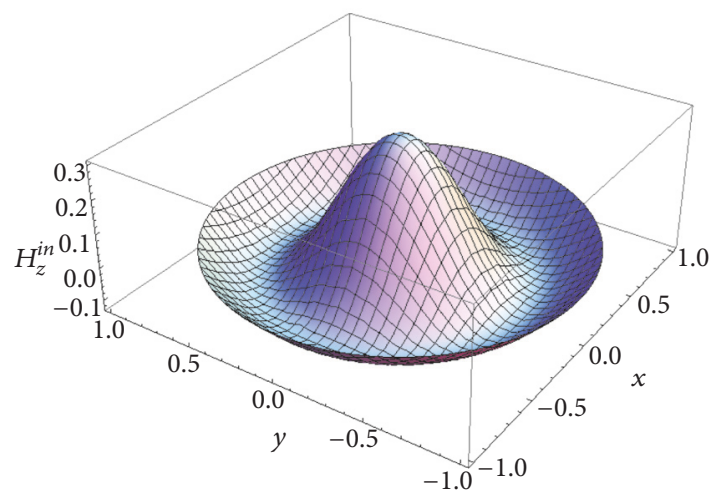

Figure 3: Plot of the magnetic field $H_{z}^{i n}(r, t)$ (see (34)) as function of the radial distance from the solenoid center $r=\sqrt{x^{2}+y^{2}}$. The parameters $I_{0}$ and $a^{*}$ have been set equal to the unit, while the field is evaluated at $\omega_{0} t=\pi / 4$ and $\omega_{0}=5.5201$, i.e., the second zero of $J_{0}(z)$. At this frequency the magnetic field vanishes on the solenoid surface.

where, exploiting the symmetries of the integral, we define the function $G(t)$ as

$$
\begin{aligned}
& G(t) \\
& \quad=\int_{0}^{\infty} \omega\left[Y_{1}\left(\omega a^{*}\right) \cos \omega t-J_{1}\left(\omega a^{*}\right) \sin \omega t\right] \frac{d \omega}{\pi} .
\end{aligned}
$$

The integral can be solved analytically [29] and gives

$$
G(t)=-\frac{2}{\pi a^{*}} \frac{\partial}{\partial t}\left[\frac{t}{\sqrt{t^{2}-a^{* 2}}} \theta\left(t-a^{*}\right)\right]
$$

where $\theta(z)$ is the step function. If $I\left(t-t^{\prime}\right)$ admits a finite limit for $t^{\prime} \longrightarrow \infty$ we can integrate by parts (48); otherwise we 


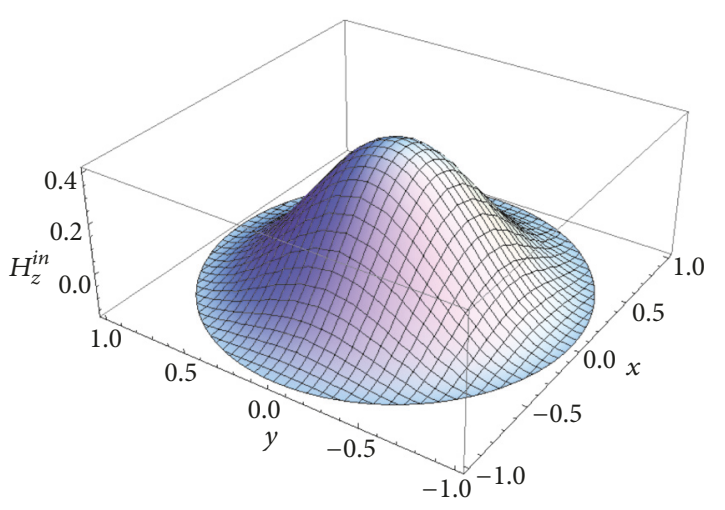

Figure 4: Plot of the magnetic field $H_{z}^{i n}(r, t)$ (see (34)) as function of the radial distance from the solenoid center $r=\sqrt{x^{2}+y^{2}}$. The parameters $I_{0}$ and $a^{*}$ have been set equal to the unit, while the field is evaluated at $\omega_{0} t=\pi / 4$ with $\omega_{0}=3.8320$, i.e., the first zero of $J_{1}(z)$. At this frequency, both the magnetic and electric fields vanish outside of the solenoid.

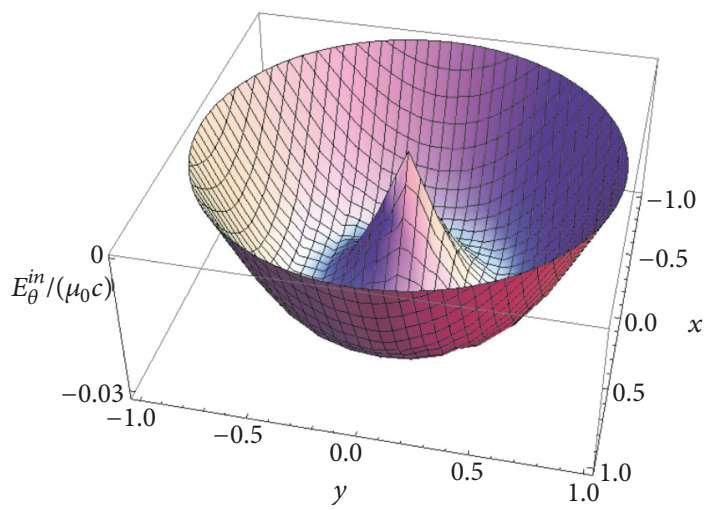

FIGURE 5: Plot of $E_{\theta}^{i n}(r, t) /\left(\mu_{0} c\right)$ (see (35)) as function of the radial distance from the solenoid center $r=\sqrt{x^{2}+y^{2}}$. The parameters $I_{0}$ and $a^{*}$ have been set equal to the unit, while the field is evaluated at $\omega_{0} t=\pi / 4$ with $\omega_{0}=3.8320$, i.e., the first zero of $J_{1}(z)$. At this frequency, both the magnetic and electric fields vanish outside of the solenoid.

must consider carefully its expression. With the assumption that such a limit exists then we may rewrite (48) as

$$
\begin{aligned}
& H_{z}^{\text {in }}(0, t) \\
& \quad=I_{\infty}(t) \\
& \quad+\int_{-\infty}^{\infty} I^{\prime}\left(t-t^{\prime}\right) \frac{t^{\prime}}{\sqrt{t^{\prime 2}-a^{* 2}}} \theta\left(t^{\prime}-a^{*}\right) d t^{\prime}
\end{aligned}
$$

where $I_{\infty}(t)$ is the limit for $t^{\prime} \longrightarrow \infty$ of $I\left(t-t^{\prime}\right)$ and $I^{\prime}(z)$ is the derivative of the current $I(z)$. As first example we turn on a constant current at $t=0$, i.e.,

$$
\mathbf{J}(t)=I(t) \widehat{\boldsymbol{\theta}}=I_{0} \theta(t) \widehat{\boldsymbol{\theta}} .
$$

Applying (51) we obtain

$$
\begin{aligned}
& H_{z}^{i n}(0, t) \\
& =\left.I_{0} \theta\left(t-t^{\prime}\right) \frac{t^{\prime} \theta\left(t^{\prime}-a^{*}\right)}{\sqrt{t^{\prime 2}-a^{* 2}}}\right|_{-\infty} ^{\infty} \\
& \quad+\int_{-\infty}^{\infty} I_{0} \delta\left(t-t^{\prime}\right) \frac{t^{\prime}}{\sqrt{t^{\prime 2}-a^{* 2}}} \theta\left(t^{\prime}-a^{*}\right) d t^{\prime} \\
& =I_{0} \frac{t}{\sqrt{t^{2}-a^{* 2}}} \theta\left(t-a^{*}\right) .
\end{aligned}
$$

The two step functions make vanish the first term on the right side of (53). It is straightforward to obtain the well-known result $H_{z}^{i n}(0, t)=I_{0}$ for $t \gg a^{*}$. Next, we consider two cases where the corrections to the quasistatic approximation play an important role. For this purpose we study the case of a strong current pulse that we can approximate with a Dirac's delta, i.e.,

$$
\mathbf{J}(t)=I(t) \widehat{\boldsymbol{\theta}}=T I_{0} \delta(t-\tau) \widehat{\boldsymbol{\theta}}
$$

where $I_{0}$ is a constant with current density dimension and $T$ is a constant with the time dimension. Applying directly (48) we obtain

$$
\begin{aligned}
& H_{z}^{i n}(0, t)=T I_{0}\left[\frac{(t-\tau) \delta\left(t-\tau-a^{*}\right)}{\sqrt{(t-\tau)^{2}-a^{* 2}}}\right. \\
& \left.-\frac{a^{* 2} \theta\left(t-\tau-a^{*}\right)}{\left[(t-\tau)^{2}-a^{* 2}\right]^{3 / 2}}\right] .
\end{aligned}
$$

The first term represents the pulse arriving at the center of the solenoid while the correction shows that, after the pulse, still it is present a magnetic field decaying as $t^{-3}$. Finally we will study a solenoid where a sinusoidal current is switched on at $\mathrm{t}=0$

$$
\mathbf{J}(t)=I(t) \widehat{\boldsymbol{\theta}}=I_{0} \theta(t) \sin \omega_{0} t \widehat{\boldsymbol{\theta}}
$$

After straightforward algebra we obtain

$$
H_{z}^{\text {in }}(0, t)=\omega_{0} I_{0} \int_{a^{*}}^{t} \cos \left[\omega_{0}\left(t-t^{\prime}\right)\right] \frac{t^{\prime}}{\sqrt{t^{\prime 2}-a^{* 2}}} d t^{\prime} .
$$

In the asymptotic limit, $t \longrightarrow \infty$, we have

$$
\begin{aligned}
& H_{z}^{i n}(0, t) \approx-\frac{\pi \omega_{0} a^{*} I_{0}}{2}\left[J_{1}\left(\omega_{0} a^{*}\right) \cos \left(\omega_{0} t\right)\right. \\
& \left.\quad+Y_{1}\left(\omega_{0} a^{*}\right) \sin \left(\omega_{0} t\right)\right] .
\end{aligned}
$$

Taking the limit for $\omega_{0} a^{*} \ll 1$ we recover the well-known quasistatic result

$$
H_{z}^{\text {in }}(0, t) \approx I_{0} \sin \left(\omega_{0} t\right)=I(t) .
$$


Taking the limit for $\omega_{0} a^{*} \gg 1$ we obtain the result

$$
H_{z}^{i n}(0, t) \approx \sqrt{\frac{\pi \omega_{0} a^{*}}{2}} I_{0} \sin \left[\omega_{0}\left(t-a^{*}\right)+\frac{\pi}{4}\right] \neq I(t) .
$$

The last two examples show that if we deal with fields that are not quasistatic, we can still consider a region where the magnetic field is uniform. On the other hand, to be consistent with Maxwell's equations we have to use the expression in (51). From expression (58) we infer that we may change the phase between flowing current and magnetic field. Selecting, for example, the frequency in such a way that $Y_{1}\left(\omega_{0} a^{*}\right)=0$ we have a magnetic field dephased of $\pi / 2$ with respect to the flowing current. In the quasistatic regime $\mathbf{H}=\mathbf{J}$ while for high frequencies the amplitude of the magnetic field grows as $\sqrt{\omega_{0}}$ and magnetic field and current density are not anymore in phase (see Figures 6 and 7). With respect to the evaluation of the electric field expression near the center of the solenoid, we may proceed in a similar way such as for the magnetic field. At the first nonvanishing order, we have

$$
\begin{gathered}
E_{\theta}^{i n}(r, t) \approx-\imath \frac{\mu_{0} c a^{*} r^{*} \pi}{2} \int_{-\infty}^{\infty} \frac{d \omega}{2 \pi} \widehat{I}_{\omega}|\omega| \\
\cdot \omega\left[Y_{1}\left(|\omega| a^{*}\right)-\imath J_{1}(\omega a)\right] \exp [-\imath \omega t] .
\end{gathered}
$$

In the time representation we have

$$
\begin{aligned}
E_{\theta}^{i n}(r, t) & =-\frac{r}{2} \frac{\partial}{\partial t} B_{z}^{i n}(0, t) \\
& =-\frac{\mu_{0} c a^{*} r^{*} \pi}{4} \int_{-\infty}^{\infty} I^{\prime}\left(t-t^{\prime}\right) G\left(t^{\prime}\right) d t^{\prime} .
\end{aligned}
$$

Formally (62) coincides with the integration in cylindrical coordinates of (5), as in the quasistatic approximation, but the magnetic field is given by (48) that in general is not proportional to the flowing current as shown by the two last examples of this section.

\section{Results and Discussion}

In this paper, we found an exact expression for the magnetic field generated by an infinite solenoid where an arbitrary current density $\mathbf{J}(t)$ is flowing. It has been shown that abrupt changes in time of the flowing current generate magnetic and electric fields that, in general, differ from the fields generated by the quasistatic approximation. This result takes in account all the corrections to the quasistatic approximation, so giving an exact expression for the uniform magnetic field generated by an arbitrary time-dependent current. Consequently, also the exact expression for the electric field associated with the time-varying magnetic field is given. The fields so found are consistent with Maxwell's equation at all time derivative order. This result can be used in several relevant applications where the current abruptly changes in time, such as pulsed current or stochastic fluctuation of the current value.

\section{Data Availability}

No data were used to support this study.

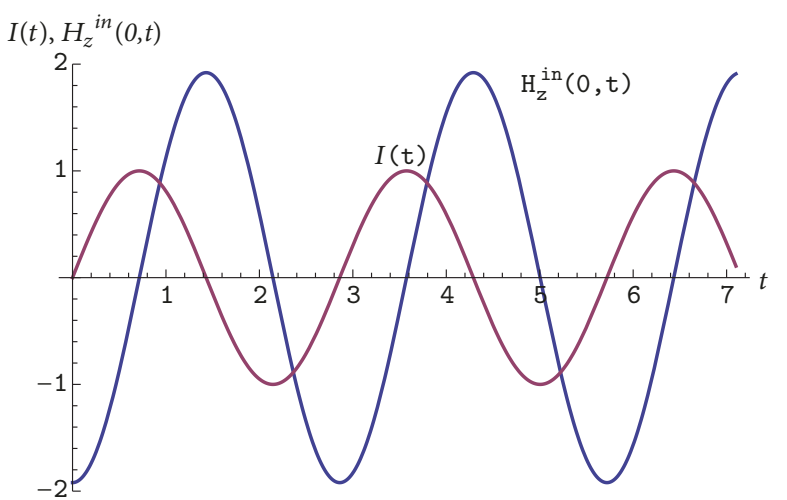

Figure 6: Plot of $I(t)=I_{0} \theta(t) \sin \omega_{0} t$, red line (see (56)), and $H_{z}^{i n}(0, t)$, blue line (see (58)) as functions of the time $t$. We set $I_{0}=1$ and $\omega_{0} a^{*}=2.1971$, i.e., the first zero of $Y_{1}(z)$. At this frequency the magnetic field and the current are dephased by $\pi / 2$.

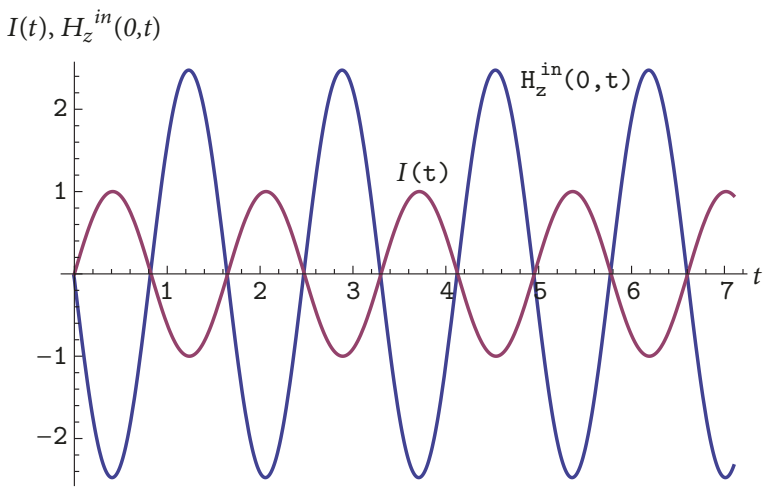

Figure 7: Plot of $I(t)=I_{0} \theta(t) \sin \omega_{0} t$, red line (see (56)), and $H_{z}^{\text {in }}(0, t)$, blue line (see (58)) as functions of the time $t$. We set $I_{0}=1$ and $\omega_{0} a^{*}=2.1971 \sqrt{3}$. At this frequency the magnetic field and the current are dephased by $\pi$.

\section{Conflicts of Interest}

The author declares that he has no conflicts of interest.

\section{Acknowledgments}

Mauro Bologna acknowledges financial support from UTA Mayor Project no. 8765-17 and the Phy.C.A for the logistical support.

\section{References}

[1] R. Merritt, C. Purcell, and G. Stroink, "Uniform magnetic field produced by three, four, and five square coils," Review of Scientific Instruments, vol. 54, no. 7, pp. 879-882, 1983.

[2] J. Dinale and J. Vrbancich, "Generation of long prolate volumes of uniform magnetic field in cylindrical saddle-shaped coils," Measurement Science and Technology, vol. 25, no. 3, p. 035903, 2014.

[3] Y. Öztürk and B. Aktas, "Generation of uniform magnetic field using a spheroidal helical coil structure," Journal of Physics: Conference Series, vol. 667, Article ID 012009, 2016. 
[4] S. R. Mahmoud, "Effect of Rotation and Magnetic Field through Porous Medium on Peristaltic Transport of a Jeffrey Fluid in Tube," Mathematical Problems in Engineering, vol. 2011, Article ID 971456, 13 pages, 2011.

[5] S. Shateyi and O. D. Makinde, "Hydromagnetic StagnationPoint Flow towards a Radially Stretching Convectively Heated Disk," Mathematical Problems in Engineering, vol. 2013, Article ID 616947, 8 pages, 2013.

[6] P. A. Bakalis, P. M. Hatzikonstantinou, and P. Vafeas, "MHD formulations for the liquid metal flow in a curved pipe of circular cross section," Computers \& Fluids, vol. 119, pp. 1-12, 2015.

[7] I. Ullah, M. T. Rahim, H. Khan, and M. Qayyum, "Analytic Comparison of MHD Squeezing Flow in Porous Medium with Slip Condition," Physics Research International, vol. 78, no. 2, pp. 1223-7027, 2016.

[8] S. Munawar, "Swirling Flow in a Permeable Tube at Slowly Expanding and Contracting Wall," Mathematical Problems in Engineering, vol. 2017, Article ID 6904161, 8 pages, 2017.

[9] P. K. Papadopoulos, P. Vafeas, and P. M. Hatzikonstantinou, "Ferrofluid pipe flow under the influence of the magnetic field of a cylindrical coil," Physics of Fluids, vol. 24, no. 12, 2012.

[10] P. Vafeas, P. K. Papadopoulos, and P. M. Hatzikonstantinou, "Analytical integro-differential representation of flow fields for the micropolar Stokes flow of a conducting ferrofluid," IMA Journal of Applied Mathematics, vol. 80, no. 3, pp. 839-864, 2015.

[11] P. Vafeas, "On the integro-differential general solution for the unsteady micropolar Stokes flow of a conducting ferrofluid," Quarterly of Applied Mathematics, vol. 76, no. 1, pp. 19-37, 2018.

[12] F. Bonetto, E. Anoardo, and M. Polello, "Saddle coils for uniform static magnetic field generation in NMR experiments," Concepts in Magnetic Resonance, vol. 29, no. 1, pp. 9-19, 2006.

[13] B. J. Gireesha, G. S. Roopa, and C. S. Bagewadi, "Unsteady Flow and Heat Transfer of a Dusty Fluid through a Rectangular Channel," Mathematical Problems in Engineering, vol. 2010, Article ID 898720, 17 pages, 2010.

[14] P. Vafeas, P. K. Papadopoulos, and P. M. Hatzikonstantinou, "On the Perturbation of the Three-Dimensional Stokes Flow of Micropolar Fluids by a Constant Uniform Magnetic Field in a Circular Cylinder," Mathematical Problems in Engineering, vol. 2011, Article ID 659691, 41 pages, 2011.

[15] A. Bakhshizadeh, M. Zamani Nejad, and M. Davoudi Kashkoli, "Time-Dependent Hygro-Thermal Creep Analysis of Pressurized FGM Rotating Thick Cylindrical Shells Subjected to Uniform Magnetic Field," Journal of Solid Mechanics, vol. 9, no. 3, pp. 663-679, 2017.

[16] Y. Xiang, Y. Chen, Q.-Z. Chen, J. Zhang, and Y.-K. Liu, “The effect of magnetic field on the susceptibility maximum in the spatially anisotropic Heisenberg antiferromagnet," Solid State Communications, vol. 148, no. 9-10, pp. 369-373, 2008.

[17] B. Tellini, M. Bologna, and A. Petri, "Measurement of magnetism in composite materials," IEEE Transactions on Instrumentation and Measurement, vol. 58, no. 10, pp. 3411-3417, 2009.

[18] M. Bologna, A. Petri, B. Tellini, and C. Zappacosta, "Effective magnetic permeability measurement in composite resonator structures," IEEE Transactions on Instrumentation and Measurement, vol. 59, no. 5, pp. 1200-1206, 2010.

[19] O. Olendski, "A charged particle in a time-varying magnetic field," Journal of Physics A: Mathematical and General, vol. 26, no. 24, pp. 7651-7661, 1993.

[20] E. R. Javor and T. Anderson, "Design of a Helmholtz coil for low frequency magnetic field susceptibility testing," in Proceedings of the 1998 IEEE International Symposium on Electromagnetic Compatibility. Part 1 (of 2), pp. 912-917, August 1998.

[21] M. Calvo and P. Paola, "Theory of a time dependent magnetic field lens," Optik (Jena), vol. 113, no. 1, pp. 31-34, 2002.

[22] S. Ray, M. Rano, and B. C. Bag, "Resonance behavior of a charged particle in presence of a time dependent magnetic field," The Journal of Chemical Physics, vol. 142, no. 15, p. 154122, 2015.

[23] L. D. Landau, E. M. Lifshitz, and L. P. Pitaevskii, Electrodynamics of Continuous Media, chapter 3, Pergamon Press, 2nd edition, 1984.

[24] J. D. Jackson, Classical Electrodynamics, Wiley, New York, NY, USA, 1962.

[25] J. D. Templin, "Exact solution to the field equations in the case of an ideal, infinite solenoid," American Journal of Physics, vol. 63, no. 10, pp. 916-920, 1995.

[26] K. T. McDonald, "The fields outside a long solenoid with a timedependent current," American Journal of Physics, vol. 65, no. 12, pp. 1176-1180, 1997.

[27] D. Bell, "Fluctuations of electric current," Journal of the Institution of Electrical Engineers - Part III: Radio and Communication Engineering, vol. 93, no. 21, pp. 37-44, 1946.

[28] G. N. Watson, A Treatise on the Theory of Bessel Functions, Cambridge University Press, Cambridge, UK, 2nd edition, 1944.

[29] I. S. Gradshteyn and I. M. Ryzhik, Table of Integrals, Series, and Products, Academic Press, 2007. 


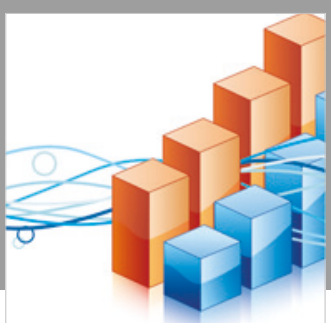

Advances in

Operations Research

\section{-n-m}
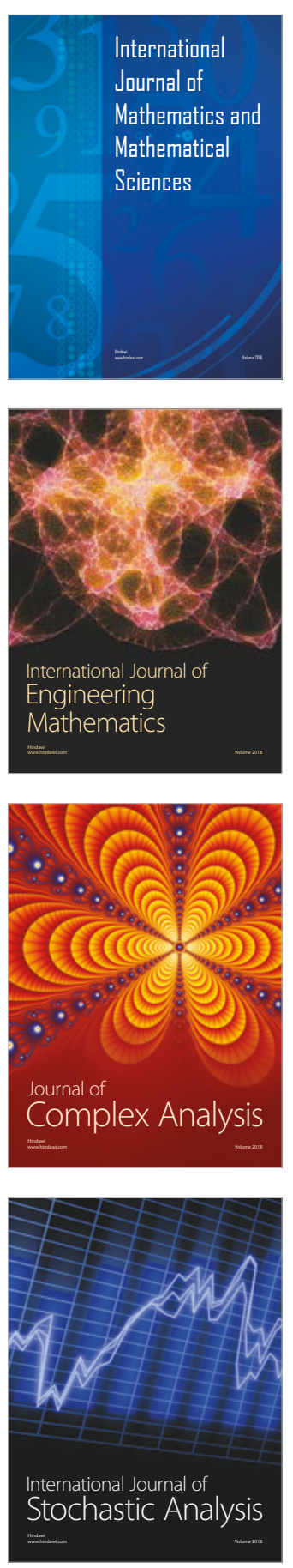
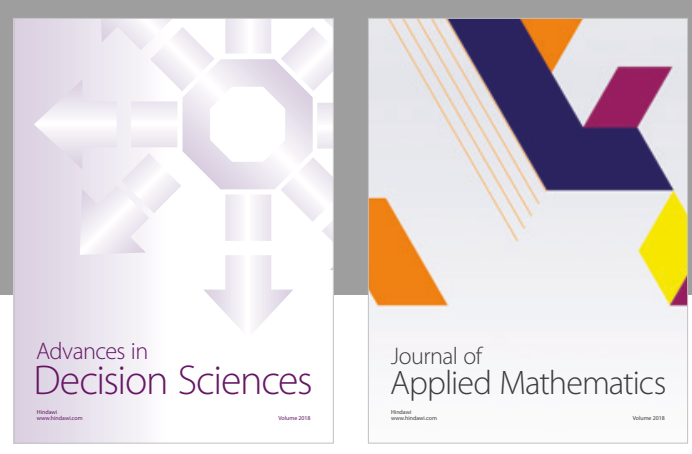

Journal of

Applied Mathematics
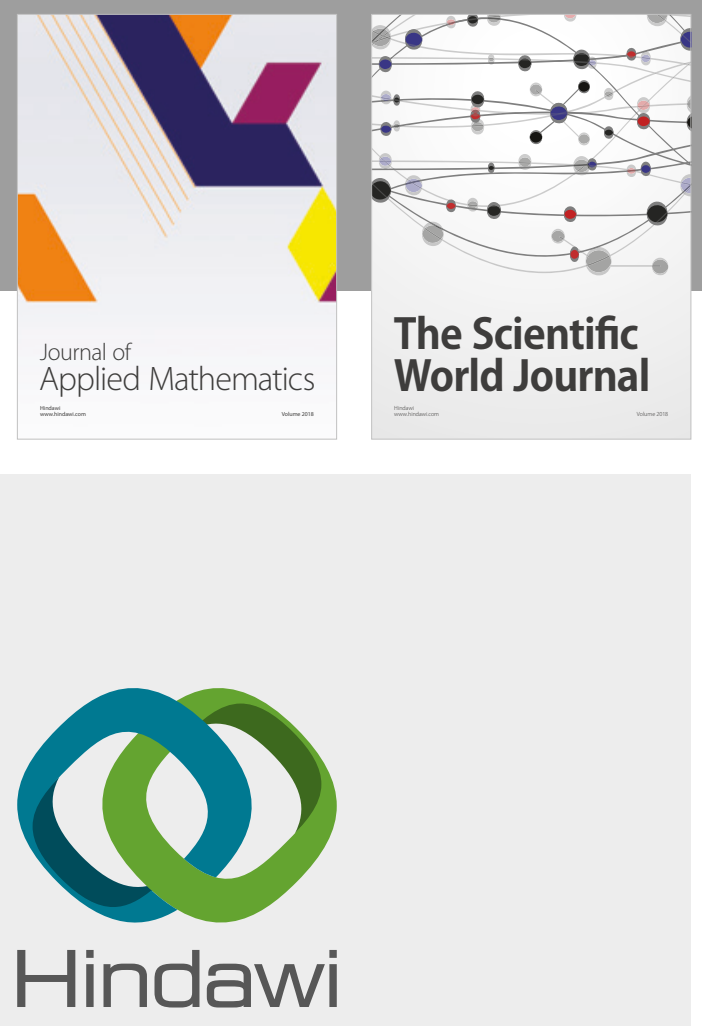

Submit your manuscripts at

www.hindawi.com

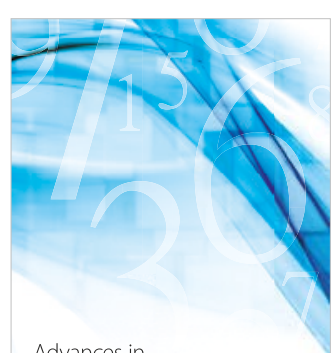

Advances in
Numerical Analysis
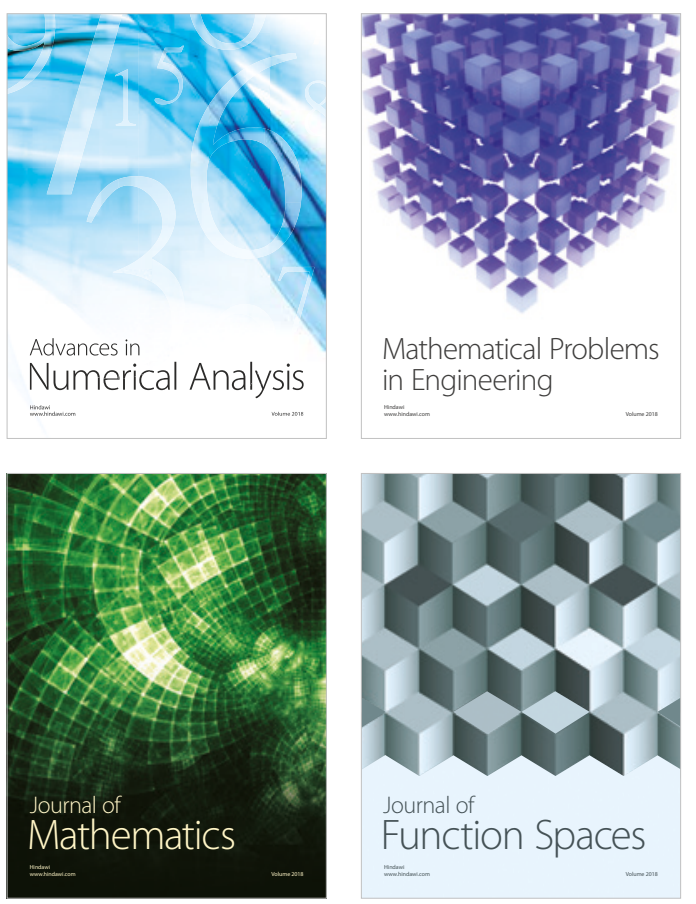

Mathematical Problems in Engineering

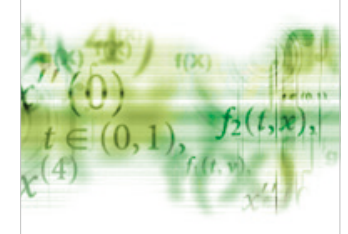

International Journal of

Differential Equations

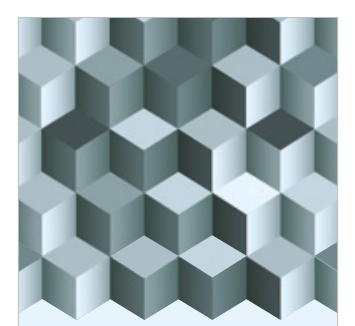

Journal of

Function Spaces

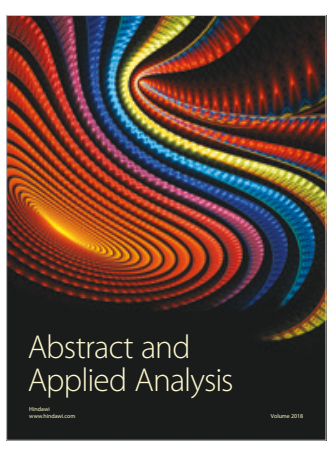

The Scientific

World Journal

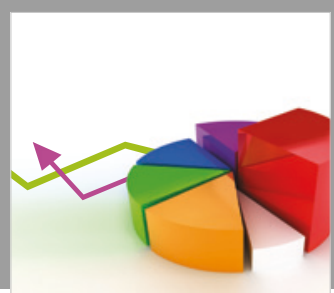

Journal of

Probability and Statistics
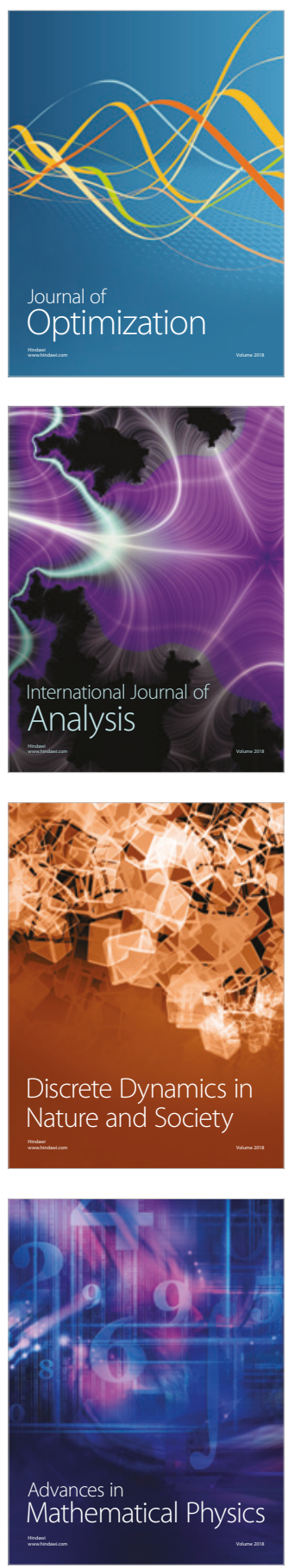